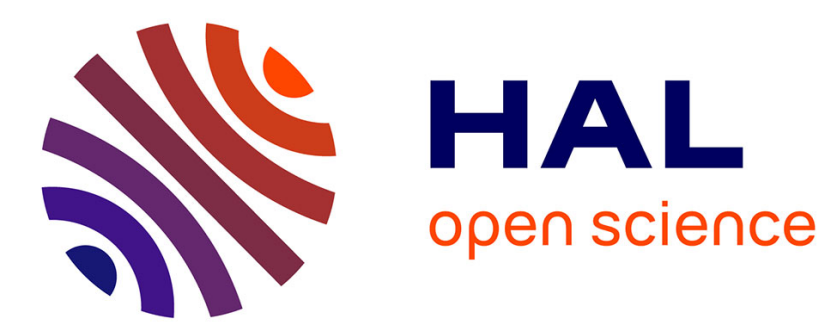

\title{
Efficacité de la coccinelle aphidiphage Semiadalia undecimnotata Schn. en serre
}

André Ferran, Marie-Madeleine Larroque

\section{To cite this version:}

André Ferran, Marie-Madeleine Larroque. Efficacité de la coccinelle aphidiphage Semiadalia undecimnotata Schn. en serre. Agronomie, 1984, 4 (4), pp.367-376. hal-00884647

\section{HAL Id: hal-00884647 \\ https://hal.science/hal-00884647}

Submitted on 1 Jan 1984

HAL is a multi-disciplinary open access archive for the deposit and dissemination of scientific research documents, whether they are published or not. The documents may come from teaching and research institutions in France or abroad, or from public or private research centers.
L'archive ouverte pluridisciplinaire HAL, est destinée au dépôt et à la diffusion de documents scientifiques de niveau recherche, publiés ou non, émanant des établissements d'enseignement et de recherche français ou étrangers, des laboratoires publics ou privés. 


\section{Efficacité de la coccinelle aphidiphage Semiadalia unde- cimnotata Schn. en serre}

André FERRAN \& Marie-Madeleine LARROQUE

I.N.R.A., Station de Zoologie et de Lutte biologique, 37, Boulevard du Cap, F 06602 Antibes

Mots clés additionnels : Prédateur, puceron, aubergine, lutte biologique, traitement inondatif.

Last-instar larvae of Semiadalia undecimnotata were used in inundative releases against eggplant aphids in the glasshouse. The number of larvae placed on each plant was determined according to the feeding rate of this instar and the total biomass of aphids. In most cases, these biological treatments induced a very appreciable decrease of aphid populations. In spite of these hopeful results, the method seems difficult to apply in large glasshouses. The main reasons for this are : the reproductive potential of the aphids, the need for a large number of larvae, the necessity for a minimum prey density in order to maintain the larvae on the plant, their prefered localization on the middle level of the eggplants, and the larval and adult mobility which results in a wastage of the biological agent. The problems which the adult mobility of this predator presents for its acclimatization in glasshouses through the crop growing period can perhaps be resolved by searching for better adapted exotic species or by selecting for less mobile strains.

Additional key words : Predator, aphid, eggplant, biological control, inundative release.

\section{INTRODUCTION}

En région méditerranéenne, le tétranyque Tetranychus urticae Koch., l'aleurode Trialeurodes vaporarorium West. et divers pucerons, Aphis gossypii Glov., Aulacorthum solani Kltb., Macrosiphum euphorbiae Thom. et Myzus persicae Sulz. sont les ravageurs les plus communs des plantes cultivées en serre.

Les deux premières espèces sont combattues à l'aide d'auxiliaires introduits : respectivement, l'acarien prédateur Phytoseiulus persimilis Athias-Henriot (HUSSEY et al., 1969 ; PARR, 1972 ; PRALAVORIO et al., 1975 ; PralaVorio, 1978) et l'hyménoptère parasite Encarsia formosa Gahan (HUSSEY \& BRAVENBOER, 1971 ; ONILLON, 1978). Par contre, la lutte contre les pucerons est encore essentiellement chimique. Cette situation résulte, à la fois, des aptitudes biologiques de ces phytophages et de certaines particularités de leurs ennemis naturels. Les pucerons, par leur précocité saisonnière, par la diversité des plantes cultivées ou spontanées qu'ils sont susceptibles de coloniser, par le nombre élevé de générations et par leurs potentialités reproductrices exceptionnelles dues principalement à leur multiplication parthénogénétique, sont capables, dans des conditions thermiques favorables, de développer des pullulations importantes dans un laps de temps très court, de l'ordre de 1 à 2 mois.

Les parasites et les prédateurs qui fréquentent naturellement le milieu «serre » et qui sont susceptibles 
de s'y multiplier sont peu nombreux. On peut citer, en particulier, les parasites Aphidius matricuriae Hal., Diaretiella rapae M'Int. et, parmi les prédateurs, la cécidomyie Aphidoletes aphidimyza Rond., les punaises Diciphus errans Wolff. et Macrolophus caliginosus Wagn., les coccinelles Propylea quatuordecimpunctata L., Adonia variegata Goeze et des Scymnus sp.. Ces auxiliaires se caractérisent par une présence sporadique et une apparition tardive lorsque les populations aphidiennes ont largement dépassé la période d'installation et même de dégâts.

De cette situation découle la nécessité d'introduire des auxiliaires dans ce milieu particulier. Malgré la richesse des espèces aphidiphages, il n'existe pas, à l'heure actuelle, d'espèce nettement adaptée à cet environnement cultural et dont l'efficacité est reconnue. Les quelques espèces qui ont fait l'objet d'applications, Aphelinus asychis Walk. et Diaretiella rapae M'Int. (LYON, 1976), Coccinella septempunctata L., Adalia bipunctata L. (HÄMÄLÄINEN, 1980), Chrysopa carnea Steph. (Tulisalo \& TuOvinen, 1975), Ch. perla (LYON, 1979), A. aphidimyza (MARKKULA et $a l ., 1979)$, sont à l'origine de résultats souvent discutables ou difficiles à généraliser sur de grandes surfaces de cultures protégées.

Dans le cadre de la recherche d'auxiliaires efficaces, nous avons utilisé les larves âgées $\left(\mathrm{L}_{4}\right)$ de la coccinelle Semiadalia undecimnotata Schn. contre les différents pucerons de l'aubergine. La connaissance des caractéristiques trophiques de cette espèce nous a permis de déterminer de manière empirique le nombre total de prédateurs à placer sur chaque plant en fonction de la population aphidienne qu'il porte. L'accent a été mis sur le contrôle de leurs déplacements car ils peuvent nous apporter des explications sur le manque d'efficacité fréquent de ce type d'entomophages.

\section{MATÉRIEL ET MÉTHODES}

Les essais de lâchers ont été réalisés dans une serre non chauffée en 1980 et 1981 , de mars à juillet.

Les aubergines, variété "Bonica》 greffées sur tomate K.N.V.F., au nombre de 20 et 24 les deux années successives, reçoivent un traitement à base de pirimicarbe dès la plantation et sont conduites selon les normes de la profession. Leur phénologie a été suivie en appliquant la méthode décrite par RABASSE (1980).
Ces plantes portent 3 espèces aphidiennes: $A$. solani, $M$. euphorbiae et $M$. persicae.

L'unité d'échantillonnage des populations aphidiennes est le plant d'aubergine dans son ensemble. Les populations aphidiennes sont exprimées en biomasse, c'est-à-dire en poids frais de pucerons. Les échantillons de pucerons sont divisés en fonction de leur taille en 5 classes : femelles ailées et aptères, larves petites, moyennes et grosses. Le poids moyen des individus de chaque classe est déterminé à partir d'individus récoltés dans la serre (tabl. 1). Le comptage, avec une périodicité hebdomadaire, du nombre total d'individus de chaque classe nous a permis de calculer, pour chaque plant et à l'issue de chaque contrôle, la biomasse relative à chaque espèce et la biomasse totale correspondante. Dans ce travail, il n'est pas tenu compte des échanges avec l'extérieur.

Les larves de la coccinelle $S$. undecimnotata utilisées au cours de ces interventions biologiques sont issues d'une population élevée en laboratoire depuis plusieurs années en présence du puceron, $M$. persicae.

Lorsque la biomasse aphidienne atteint, sur un plant, un certain niveau qui sera précisé ultérieurement, des larves âgées de $3^{\text {e }}$ stade sont placées, dès leur obtention, au sommet du végétal. Comme la mue intervient dans les heures qui suivent le lâcher, le contrôle des populations aphidiennes est assuré en fait par des individus se trouvant au dernier stade.

Le nombre de larves par plant est calculé d'une manière empirique en fonction de l'évolution dans le temps de la biomasse aphidienne totale et des aptitudes trophiques des larves de dernier stade qui ont été déterminées antérieurement (FERRAN \& LARROQUE, 1977). Le jour ( $T$ ) du traitement, on calcule le coefficient $(\mathrm{K})$ de multiplication de la biomasse aphidienne par rapport au dénombrement précédent :

$$
\mathrm{K}=\frac{\mathbf{B}_{(\mathbf{T})}}{\mathbf{B}_{(\mathbf{T}-6)}}
$$

avec $B_{(T)}$ et $B_{(T-6)}$ les biomasses aphidiennes aux jours $(\mathrm{T})$ et $(\mathrm{T}-6)$.

Si l'on suppose que ce coefficient est sensiblement constant - éventualité approximativement vérifiée lorsque les populations aphidiennes, après avoir franchi la phase d'installation, acquièrent une croissance exponentielle - il est possible d'estimer la biomasse $B_{(T+6)}$ qui serait présente sur le végétal, la semaine

TABLEAU 1

Poids moyen (en $\mathrm{mg}$ ) des pucerons constituant chaque classe de taille pour les 3 espèces qui vivent sur les aubergines en serre. Mean aphid weight (in $\mathrm{mg}$ ) of each size class for the 3 species living on eggplants in the glasshouse.

\begin{tabular}{|c|c|c|c|c|c|}
\hline \multirow{3}{*}{$\begin{array}{l}\text { Espèces } \\
\text { aphidiennes }\end{array}$} & \multicolumn{5}{|c|}{ Classes de taille } \\
\hline & \multicolumn{2}{|c|}{ Larves } & \multicolumn{3}{|c|}{ Adultes (femelles) } \\
\hline & Petites & Moyennes & Grosses & Aptères & Ailées \\
\hline Aulacorthum solani Kltb. & $\begin{array}{r}0,33 \\
0,08\end{array}$ & $\begin{array}{l}0,54 \\
0,10\end{array}$ & $\begin{array}{r}0,84 \\
0,09\end{array}$ & $\pm \begin{array}{l}1,03 \\
0,11\end{array}$ & $\begin{array}{r}1,30 \\
0,16\end{array}$ \\
\hline Macrosiphum eupnorbiae Thom. & $\begin{array}{r}0,23 \\
0,05\end{array}$ & $\pm \begin{array}{l}0,81 \\
0,10\end{array}$ & $\pm \begin{array}{r}1,40 \\
0,09\end{array}$ & $\pm \begin{array}{l}1,93 \\
0,12\end{array}$ & $\begin{array}{r}2,00 \\
0,23\end{array}$ \\
\hline Myzus persicae Sulz. & $\begin{array}{r}0,08 \\
0,02\end{array}$ & $\begin{array}{l}0,32 \\
0,06\end{array}$ & $\pm \begin{array}{l}0,49 \\
0,05\end{array}$ & $\pm \begin{array}{l}0,66 \\
0,05\end{array}$ & $\begin{array}{r}0,39 \\
\pm 0,09\end{array}$ \\
\hline
\end{tabular}


suivante, en l'absence de traitement biologique :

$$
\mathrm{B}_{(\mathrm{T}+6)}=\mathrm{KB}_{(\mathrm{T})} \text {. }
$$

Au cours du $4^{\mathrm{e}}$ stade qui dure de 4 à $6 \mathrm{j}$ pour des températures comprises entre $15^{\circ} \mathrm{C}$ et $30^{\circ} \mathrm{C}$, chacune de ces larves consomme en moyenne $70 \mathrm{mg}$ d'aphides (FERRAN \& LARROQUE, 1979).

Le nombre $(\mathrm{N})$ de larves qui doivent être placées sur un plant pour réduire la population aphidienne correspondante, est estimé, en première approximation, par la relation :

$$
N=\frac{B_{(T+6)}}{70} \text {. }
$$

En 1981, la localisation des larves sur les aubergines et leur évolution numérique ont été contrôlées chaque jour, de préférence le matin, jusqu'à leur nymphose. Les adultes qui en proviennent ont été soumis aux mêmes observations.

\section{RÉSULTATS}

\section{A. Quelques considérations sur les populations aphi- diennes}

$\mathrm{Au}$ cours de ces 2 années, les premiers pucerons sont apparus sur les aubergines environ $10 \mathrm{j}$ après le traitement insecticide général. L'infestation initiale résulte de l'arrivée de femelles ailées mais, par la suite, des formes aptères ont participé à l'extension des foyers primaires.

En 1980, M. euphorbiae était l'espèce dominante ; sa biomasse moyenne au moment des interventions représentait $89,6 \pm 4,1$ p. 100 de la biomasse totale correspondante. En $1981,91,2 \pm 3,5$ p. 100 de cette dernière était représentée par $M$. persicae., $M$. euphorbiae et $A$. solani sont apparus très tard et sont restés localisés sur un nombre réduit d'aubergines.

$\mathrm{Au}$ moment des traitements biologiques, les populations aphidiennes sont localisées sur les feuilles basales $(\mathrm{m}<1)$ repérées par rapport à la $1^{\text {re }}$ inflorescence et sur les rameaux secondaires $P 1$ et $P 2$ (fig. 1).

La croissance en biomasse des populations aphidiennes se fait selon une courbe exponentielle dans l'intervalle de temps compris entre l'infestation naturelle des plants et les dates des lâchers (tabl. 2).

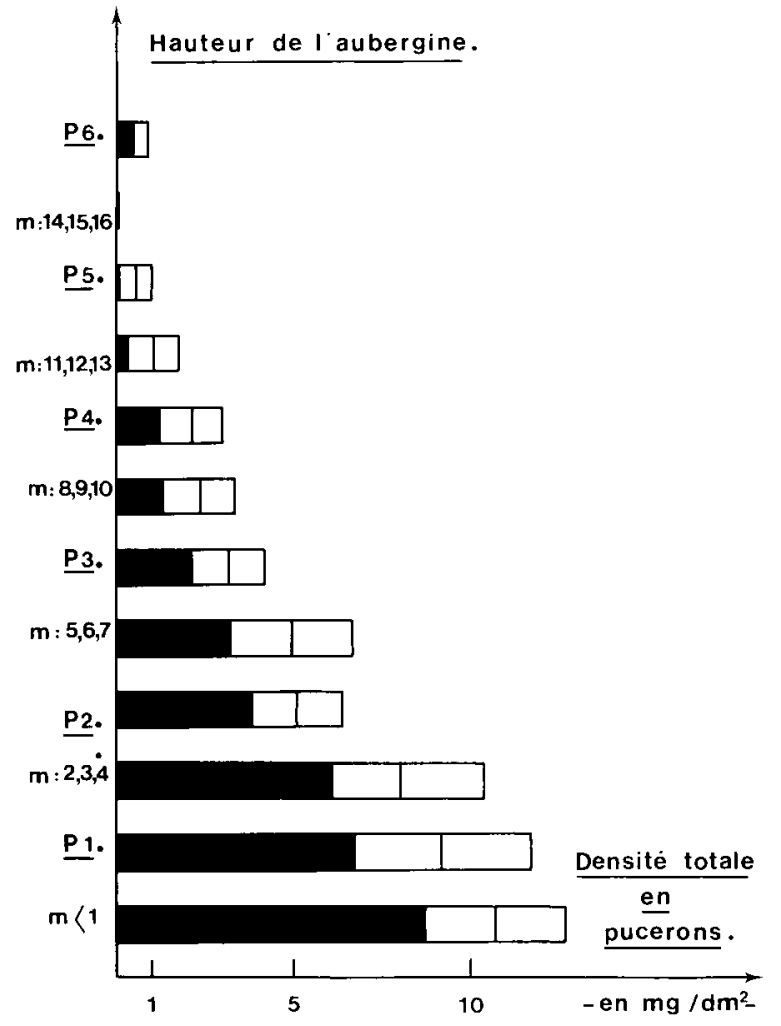

Figure 1

Répartition moyenne de la biomasse aphidienne totale sur les aubergines au moment des lâchers. Repérage des feuilles $(m)$ et des rameaux secondaires $(P)$ selon $R$ ABASSE (1980). Le trait vertical : lo moyenne-zone blanche: son intervalle de confiance $(5 \mathrm{p} .100)$.

Mean distribution of total aphid biomass on eggplants when larvae were released. Leaves $(m)$ and secondary branches $(P)$ marked according to RABASSE (1980). Vertical line mean; white zone $5 \%$ confidence interval.

\section{B. L'efficacité prédatrice des larves}

Elle est exprimée par le « taux de prédatisme apparent " (T.P.A.) qui est égal au rapport, multiplié par 100 , entre la diminution de la biomasse aphidienne totale après le traitement biologique et la biomasse observée le jour où celui-ci a été effectué :

$$
\text { T.P.A. }=\frac{B_{(T+6)}-B_{(T)}}{B_{(T)}} \times 100 .
$$

TABLEAU 2

Ajustement des populations aphidiennes à l'exponentielle $B=a e^{b t}(r$, coefficient de corrélation) dans l'intervalle de temps compris entre l'infestation naturelle des plantes et la date moyenne des interventions biologiques.

\begin{tabular}{|c|c|c|c|c|c|c|}
\hline \multirow{2}{*}{ Année } & \multicolumn{3}{|c|}{ Biomasse totale } & \multicolumn{3}{|c|}{ Biomasse du puceron dominant } \\
\hline & $a$ & $\mathbf{b}$ & r & a & $\mathrm{b}$ & $\mathbf{r}$ \\
\hline 1980 & $\pm \begin{array}{l}0,10 \\
0,01\end{array}$ & $\pm \begin{array}{l}0,24 \\
0,02\end{array}$ & $\pm \begin{array}{l}0,96 \\
0,01\end{array}$ & $\pm \begin{array}{l}0,10 \\
0,01\end{array}$ & $\pm \begin{array}{l}0,24 \\
0,02\end{array}$ & $\pm \begin{array}{l}0,95 \\
0,02\end{array}$ \\
\hline 1981 & $\begin{array}{r}0,30 \\
\pm \quad 0,19\end{array}$ & $\begin{array}{r}0,19 \\
\pm 0,03\end{array}$ & $\begin{array}{r}0,97 \\
0,01\end{array}$ & $\pm \begin{array}{l}0,16 \\
0,09\end{array}$ & $\pm \begin{array}{l}0,21 \\
0,04\end{array}$ & $\begin{array}{r}0,96 \\
0,01\end{array}$ \\
\hline
\end{tabular}

Fitting of the aphid population growth to the exponential curve $B=a e^{b t}(r$, correlation coefficient) from their natural arrival on plants to the mean date of biological treatment. 
TABLEAU 3

Année 1980. Efficacité des larves âgées de la coccinelle S. undecimnotata ( $N$ : nombre de larves par plant $-B_{(T)}$ biomasse aphidienne totale - T.P.A. : taux de prédatisme apparent).

Year 1980. Efficiency of old larvae of $\mathrm{S}$. undecimnotata $\left(N\right.$ : number of larvae per plant $-B_{(T)}$ : total aphid biomass - TPA : apparent predatory ratio).

\begin{tabular}{|c|c|c|c|c|c|}
\hline $\mathrm{N}^{\circ}$ du plant & Dates des lâchers & $\mathbf{N}$ & $\mathrm{B}_{(\mathrm{T})}$ & T.P.A. & Nombre de nymphes \\
\hline \multirow[t]{3}{*}{2} & 12,6 & 26 & 329 & - & 0 \\
\hline & 23,6 & 50 & 1290 & 34,7 & 11 \\
\hline & 1,7 & 15 & 843 & 16,5 & 23 \\
\hline 3 & 23,6 & 15 & 831 & 52,0 & 24 \\
\hline \multirow[t]{2}{*}{4} & 12,6 & 44 & 871 & 83,7 & 28 \\
\hline & 1,7 & 42 & 887 & 76,9 & 42 \\
\hline \multirow[t]{2}{*}{5} & 12,6 & 24 & 440 & 67,8 & 9 \\
\hline & 1,7 & 71 & 1055 & 96,0 & 18 \\
\hline \multirow[t]{2}{*}{6} & 12,6 & 20 & 35 & - & 4 \\
\hline & 25,6 & 50 & 1613 & 81,0 & 28 \\
\hline 7 & 23,6 & 16 & 673 & 16,7 & 5 \\
\hline \multirow{2}{*}{8} & 12,6 & 19 & 185 & - & 0 \\
\hline & 2,7 & 50 & 1961 & 60,2 & 54 \\
\hline \multirow[t]{2}{*}{9} & 12,6 & 10 & 138 & - & 0 \\
\hline & 25,6 & 100 & 2648 & 80,7 & 64 \\
\hline \multirow[t]{2}{*}{10} & 12,6 & 17 & 212 & - & 0 \\
\hline & 25,6 & 50 & 1600 & 75,1 & 13 \\
\hline \multirow[t]{2}{*}{11} & 13,6 & 10 & 170 & - & 0 \\
\hline & 27,6 & 100 & 2364 & 79,0 & 94 \\
\hline 13 & 27,6 & 50 & 886 & 62,0 & 17 \\
\hline 14 & 27,6 & 40 & 971 & 79,5 & 19 \\
\hline 15 & 27,6 & 16 & 745 & 69,9 & 15 \\
\hline 16 & 17,6 & 15 & 1053 & 98,8 & 7 \\
\hline \multirow[t]{2}{*}{17} & 17,6 & 15 & 541 & 50,8 & 10 \\
\hline & 3,7 & 90 & 1356 & 69,6 & 15 \\
\hline 18 & 30,6 & 100 & 1408 & 89,0 & 24 \\
\hline 22 & 4,7 & 80 & 1307 & 91,6 & 10 \\
\hline
\end{tabular}

Afin de limiter la taille de l'inoculum larvaire, 6 lâchers (plants $2,6,8,9,10,11$ ) ont été réalisés en 1980 pour des infestations aphidiennes inférieures à $400 \mathrm{mg}$ (tabl. 3). Dans tous les cas, les larves ont quitté les aubergines correspondantes sans affecter notablement les populations des proies. Grâce à des lâchers mis en place à différents niveaux d'infestation, nous avons estimé de 400 à $500 \mathrm{mg}$ la quantité minimale de pucerons qu'une aubergine doit porter pour que les larves fassent preuve d'une efficacité mesurable. Ces valeurs sont sensiblement équivalentes à $1000 \mathrm{M}$. euphorbiae ou à $2300 \mathrm{M}$. persicae dans une population de structure démographique normale. Par la suite, les lâchers ont été réalisés lorsque la biomasse aphidienne totale dépassait ce seuil.

En 1980, sur les 21 interventions biologiques effectuées, 3 ont donné des T.P.A. inférieurs à 50 p. 100, 2 des T.P.A. compris entre 50 et 60 p. 100 et 16 des T.P.A. supérieurs à $60 \mathrm{p}$. 1010 . Sur certains plants $(5,16,18,22)$, les populations aphidiennes ont été pratiquement éliminées.

En 1981, les résultats ont été meilleurs puisque 3 T.P.A. seulement ont été inférieurs à 60 p. 100 tandis que 11 ont été supérieurs à 80 p. 100 (tabl. 4). Cette amélioration sensible de l'efficacité peut résulter de la différence de taille entre $M$. persicae et $M$. euphorbiae : les larves doivent consommer un plus grand nombre d'individus de l'espèce la plus petite pour couvrir leurs besoins de croissance. Elle peut être rapprochée également du fait que le puceron dominant dans la culture ( $M$. persicae) est utilisé pour l'élevage permanent de cette coccinelle en labo- ratoire. Cette dernière hypothèse semble toutefois contredire l'opinion de MURDOCK \& MARKS (1973) selon laquelle ces prédateurs ne peuvent pas être conditionnés à une proie particulière.

A quelques exceptions près, cette méthode de lutte biologique nous a permis de stopper l'évolution exponentielle des populations aphidiennes et même, dans de nombreux cas, de les réduire très substantiellement (fig. 2, 3, 4, 5).

Toutefois l'équilibre «proie-prédateur » ainsi créé est temporaire. Sur tous les plants traités, nous avons observé l'amorce de nouvelles pullulations liées au puceron dominant ou à l'une des 2 autres espèces (fig. 2). Elles nous auraient probablement imposé de nouveaux traitements si ces travaux n'avaient pas été arrêtés le $1^{\text {er }}$ juillet, à moins que les conditions thermiques qui règnent dans les serres pendant la période estivale et le vieillissement des végétaux ne limitent leur importance (LYON, 1976).

De mars à juillet, un traitement insecticide effectué dès la plantation puis un lâcher de larves nous ont permis de limiter les populations aphidiennes, exception faite des plants $2,4,5,6,8,9,10,11$ en 1980 et des plants 5,7 en 1981 qui ont subi 2 ou même 3 interventions biologiques (tabl. 3 et 4 ).

\section{Le comportement larvaire}

Les larves de cette coccinelle consomment les 3 espèces aphidiennes précitées bien que 2 d'entre elles, $A$. solani et $M$. euphorbiae, ne paraissent pas être des proies habituelles. 
TABLEAU 4

Année 1981. Efficacité des larves âgées de la coccinelle $\mathrm{S}$. undecimnotata ( $N$ : nombre de larves par plant $-B_{(T)}$ : biomasse aphidienne totale - T.P.A. : taux de prédatisme apparent).

Year 1981. Efficiency of old larvae of $\mathrm{S}$. undecimnotata $\left(N\right.$ : number of larvae per plant $-B_{(T)}$ : total aphid biomass - TPA : apparent predatory ratio).

\begin{tabular}{|c|c|c|c|c|c|}
\hline $\mathrm{N}^{\circ} \mathrm{du}$ plant & Dates des lâchers & $\mathrm{N}$ & $\mathrm{B}_{(\mathrm{T})}$ & T.P.A. & Nombre de nymphes \\
\hline 1 & 9,6 & 60 & 1070 & 85,4 & 29 \\
\hline 2 & 9,6 & 170 & 1835 & 85,2 & 16 \\
\hline 4 & 22,6 & 50 & 723 & 48,3 & 13 \\
\hline \multirow[t]{2}{*}{5} & 15,6 & 50 & 1599 & 57,3 & 26 \\
\hline & 29,6 & 75 & 1893 & 85,2 & 28 \\
\hline 6 & 29,6 & 50 & 1000 & 66,4 & 14 \\
\hline \multirow[t]{2}{*}{7} & 1,6 & 50 & 693 & 63,6 & 20 \\
\hline & 23,6 & 50 & 762 & 18,2 & 30 \\
\hline 8 & 1,6 & 105 & 2022 & 90,0 & 65 \\
\hline 9 & 10,6 & 90 & 1590 & 73,0 & 26 \\
\hline 10 & 18,5 & 65 & 863 & 97,9 & 32 \\
\hline 11 & 18,5 & 155 & 1008 & 95,9 & 34 \\
\hline 12 & 19,5 & 110 & 1035 & 96,6 & 11 \\
\hline 15 & 3,6 & 55 & 1298 & 90,7 & 30 \\
\hline 16 & 3,6 & 44 & 746 & 78,9 & 18 \\
\hline 19 & 24,6 & 80 & 1182 & 80,2 & 38 \\
\hline 20 & 19,5 & 80 & 700 & 94,5 & 9 \\
\hline 21 & 25,6 & 80 & 1343 & 76,9 & 29 \\
\hline 22 & 25,6 & 100 & 1622 & 82,0 & 47 \\
\hline 23 & 26,6 & 80 & 1305 & 76,9 & 36 \\
\hline 24 & 29.6 & 75 & 884 & 62,1 & 57 \\
\hline
\end{tabular}

La comparaison des effectifs respectifs des différentes espèces et même des différentes classes au sein de chaque espèce nous a permis de montrer que les larves attaquent les groupes qui sont numériquement dominants. Chez les coccinelles, le choix d'une proie ne dépend pas de sa valeur alimentaire mais de la fréquence des contacts, car la recherche des pucerons se fait au hasard (BLACKMAN, 1967).

Les larves qui ont été déposées au sommet des plants, en fin de journée, se répartissent sur l'ensemble du feuillage en $15 \mathrm{~h}$ environ. Passé ce délai, nous avons constaté que le nombre de larves sur les feuilles basses $(m<1)$ est comparable (malgré la présence de fortes infestations aphidiennes) à celui observé sur les feuilles terminales $(\mathrm{m}>10)$. Cette fréquentation limitée des feuilles basales qui sont souvent au contact du sol, a été également observée chez $A$. bipunctata par HÄMÄLÄINEN (1977).

Le nombre de larves par plant diminue très rapidement dans les 2 premiers jours après le lâcher puis se stabilise peu avant la nymphose (fig. 6). HÄMÄLÄIMEN (1980) explique cette émigration par la raréfaction rapide des proies lorsque la taille de l'inoculum larvaire est élevée. La précocité de cette disparition dans nos essais permet d'envisager d'autres causes, notamment l'intervention d'un effet de nombre. Les larves qui quittent le plant traité colonisent les aubergines voisines lorsqu'elles sont suffisamment infestées ou se dispersent plus largement dans la serre, la répartition des individus avec ces deux possibilités étant en général comparable (fig. 7). Les larves peuvent quitter les végétaux accidentellement (chute) ou par l'intermédiaire des tiges ou des feuilles qui touchent la terre. Bien que, dans cette situation, leur chance de trouver une nouvelle plante soit faible, elles peuvent survivre et même se nymphoser en raison de la présence de proies sur le sol et de leur résistance à des conditions trophiques défavorables.

\section{La mobilité des adultes}

Dans un délai approximatif de 2 à $5 \mathrm{j}$ après la mue imaginale, les adultes, mâles et femelles, quittent la serre par les ouvrants d'aération. Ces départs interviennent essentiellement aux heures chaudes de la journée (fig. 8).

Cette mobilité des adultes paraît très générale chez les coccinelles. Elle a été observée aussi bien en serre qu'en plein air (GURNEY \& HUSSEY, 1970 ; HÄMÄLÄINEN, 1980 ; IVES, 1981 ; KIECKHEFER \& OLSON, 1974 ; TAMAKI \& WEEKS, 1973). Ses causes paraissent multiples. La localisation préférentielle des adultes sur les parties hautes de la plante, l'existence d'une densité minimale en proies pour leur maintien sur les végétaux au printemps (FRAZER et al., 1981), les placent dans des conditions trophiques défavorables lorsque l'essentiel de l'activité prédatrice est confié aux stades immatures. L'existence d'une mobilité intrinsèque maximale liée à l'immaturité des jeunes femelles et dépendante des conditions thermiques ambiantes est généralement admise (IPERTI, comm. pers.). Enfin, l'apparition très localisée dans le temps et dans l'espace d'un grand nombre d'adultes pourrait être à l'origine d'interférences qui provoqueraient leur dispersion (HASSELL et al., 1976).

Au cours de ces 2 années, des adultes dans l'incapacité de voler en raison de malformations alaires se sont maintenus dans la serre, se sont accouplés et ont donné naissance à une $2^{\mathrm{e}}$ génération larvaire.

\section{E. Présence d'auxiliaires spontanés}

Au cours de l'année 1981, nous avons compté, une fois par semaine et sur chaque aubergine, le nombre de momies non perforées et le nombre de larves de cécidomyies, ces dernières nous ayant paru nombreuses l'année précédente. 


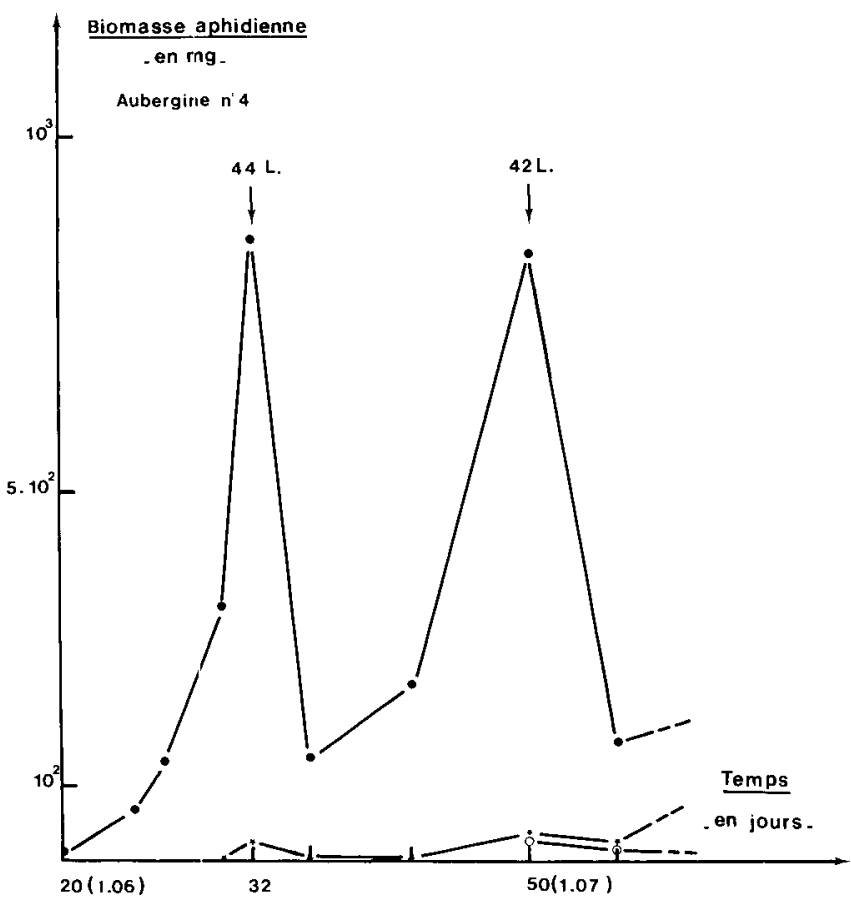

Figures $2 \& 3$

Année 1980. Variation de la biomasse aphidienne sur aubergine en serre $(\bullet:$ M. euphorbiae ; $\circ:$ A. solani ; $\star$ : M. persicae ; $n L$ : $\mathrm{S}$. undecimnotata, nombre de larves lâchées).

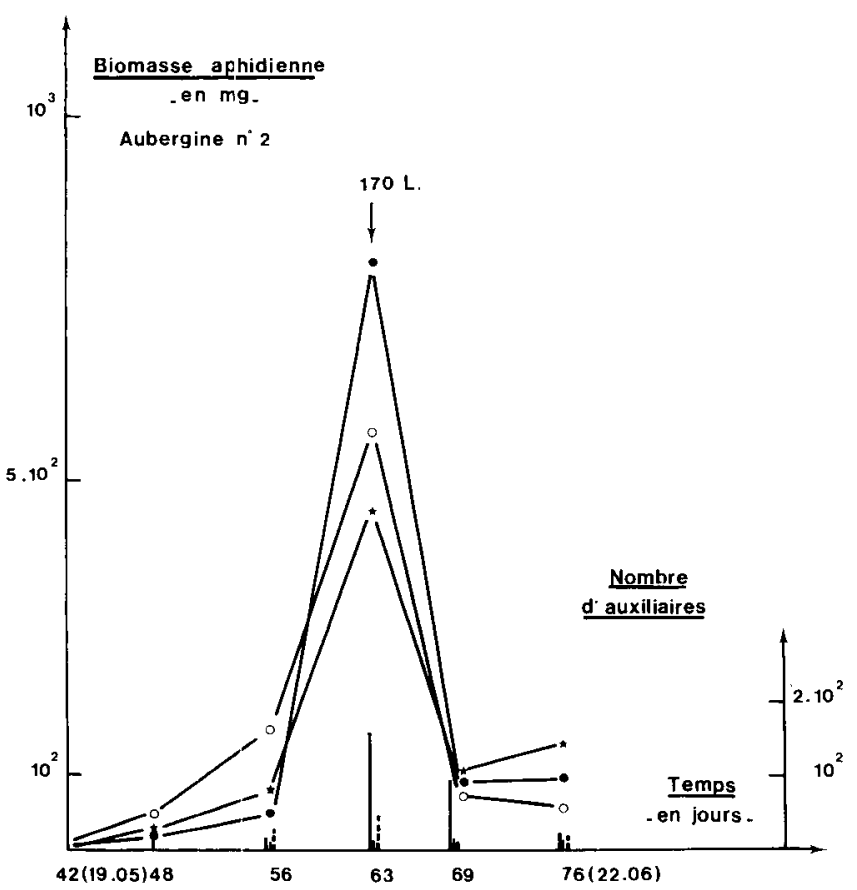

Figures 4 \& 5

Année 1981. Variation de la biomasse aphidienne sur aubergine en serre $(\bullet:$ M. euphorbiae ; 0 : A. solani ; $\star$ : M. persicae ; trait vertical continu : nombre de momies; trait vertical pointillé : nombre de larves $d$ 'A. aphidimyza (Cecidomyie); $n L$ : S. undecimnotata, nombre de larves lâchées).

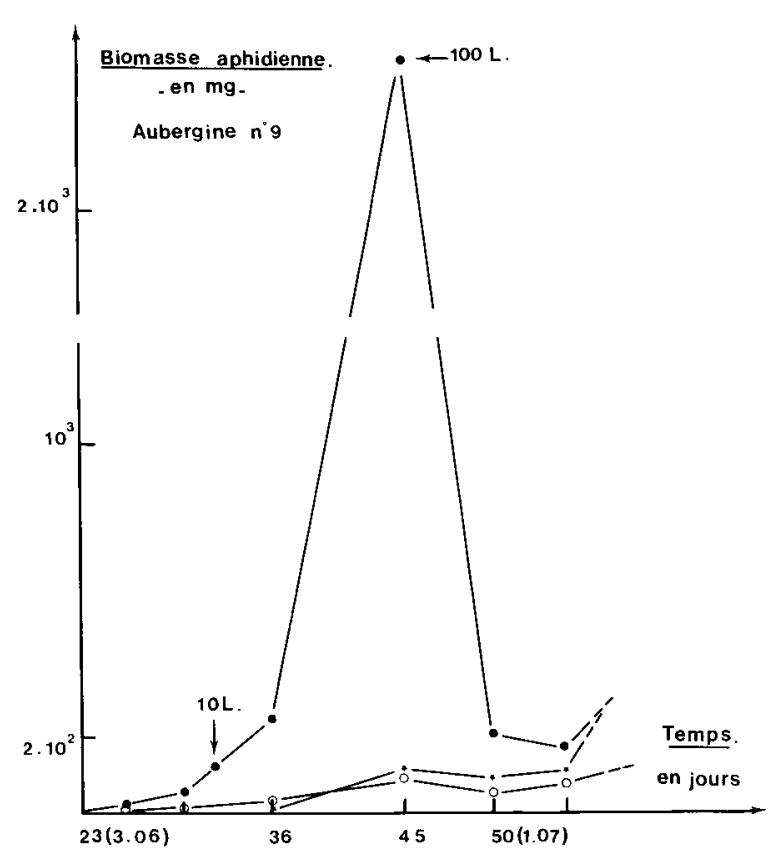

Year 1980. Change in aphid biomass on eggplants in the glasshouse $(\bullet:$ M. euphorbiae ; $\circ:$ A. solani ; $\star$ : M. persicae; $n L$ : S. undecimnotata, number of larvae released).

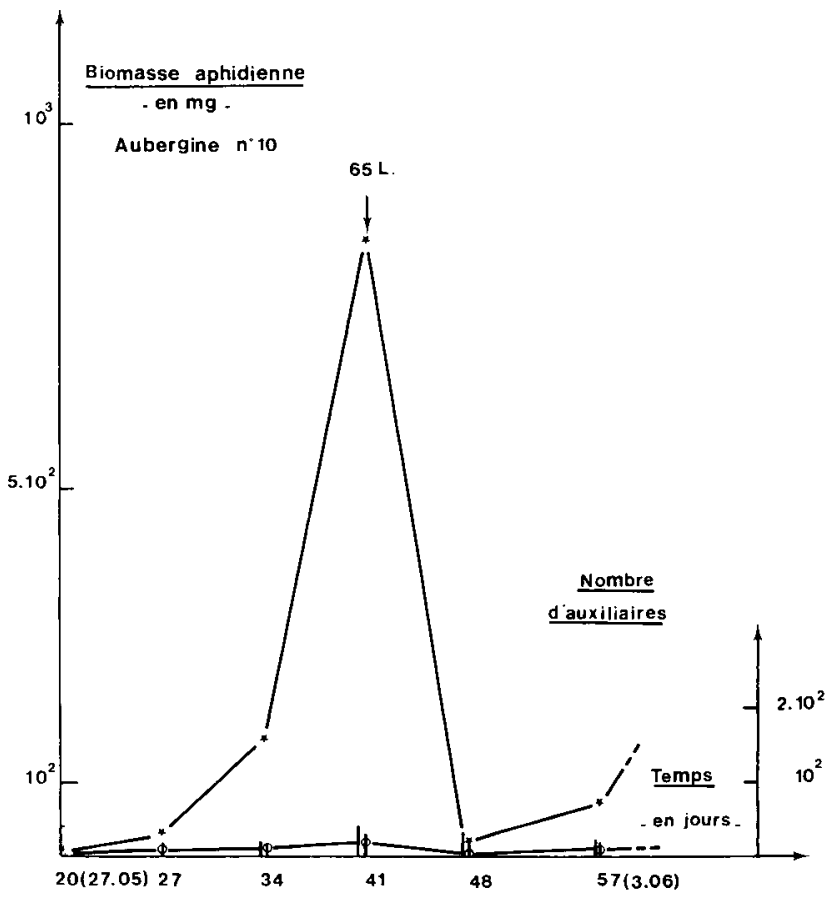

Year 1981. Change in aphid biomass on eggplants in the glasshouse $(\bullet:$ M. euphorbiae ; $\circ:$ A. solani ; $\star$ : M. persicae; vertical unbroken line: number of mummies; vertical dotted line: number of larvae of A. aphidimyza (aphid midge) ; $n L: S$. undecimnotata, number of larvae released). 


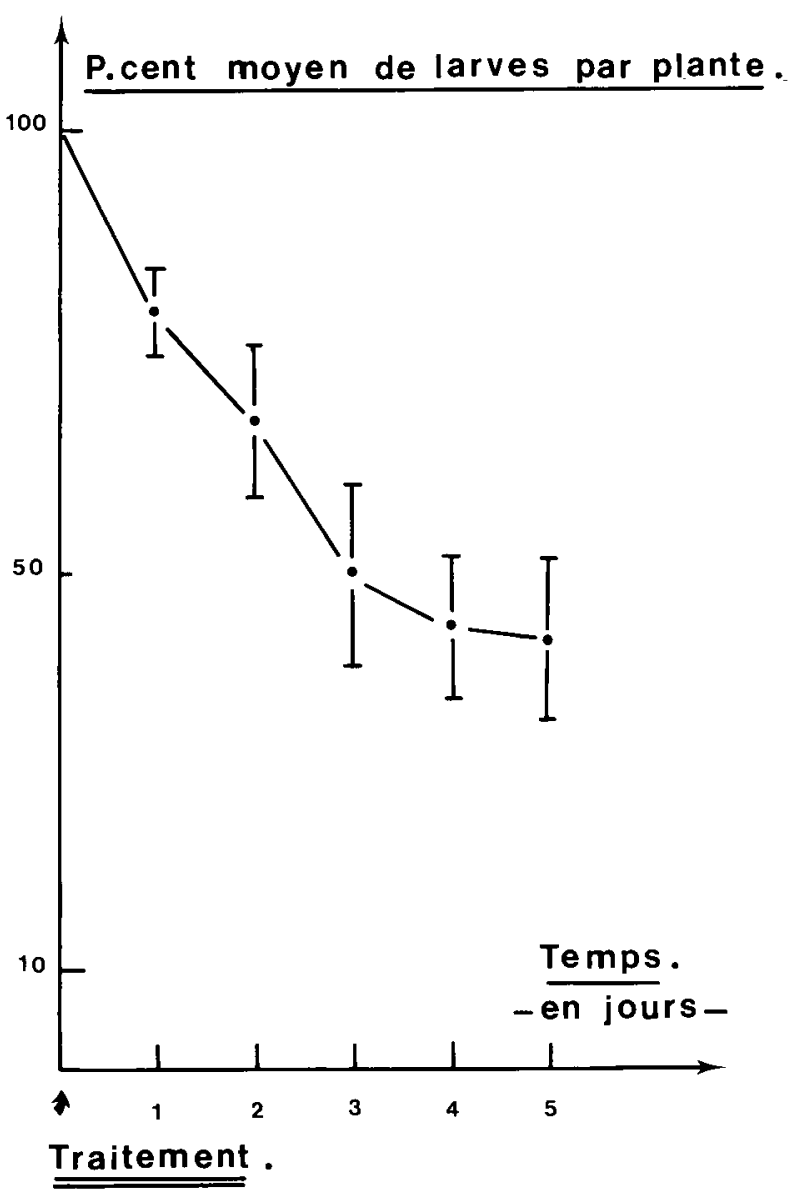

Figure 6

Variation du nombre moyen de larves après les traitements biologiques. Résultats en p. 100 par rapport au nombre de larves lâchées. Change in the mean number of larvae after the biological treatments. Results as \% of larvae released.
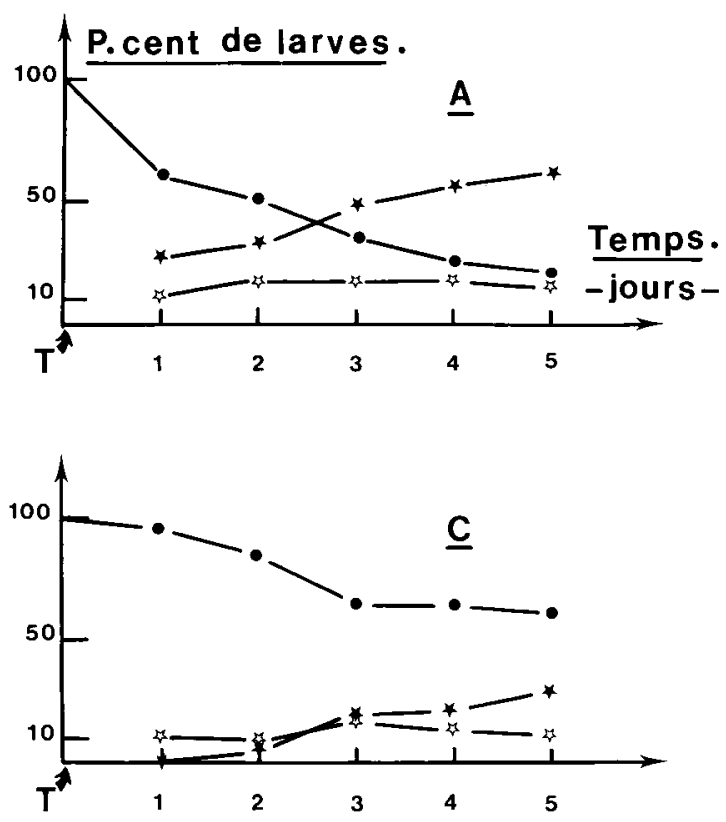

Figure 7

Variation, par rapport au nombre d'individus lâchés, de la population larvaire de $\mathrm{S}$. undecimnotata sur les aubergines traitées $(\bullet)$ et sur les aubergines voisines $(\star)$. La différence (ઐ) représente les larves disparues. $A, B, C, D$ : traitement $(T)$ biologique des auber-

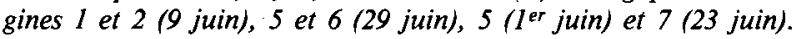
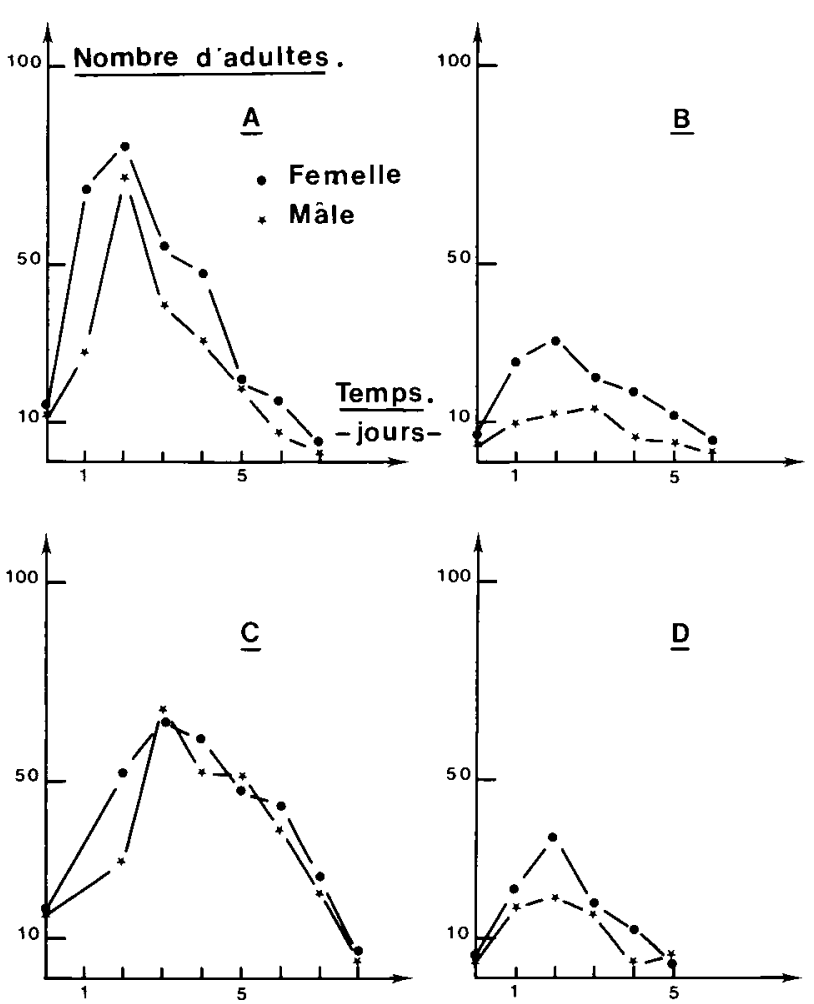

Figure 8

Variation du nombre total d'adultes issus des larves lâchées. $A, B$, $C, D$ : aubergines $10,11,12$ et 20 (18 et 19 mai), I er et 2 (9 juin), $4,5,7,9,19,21$ et 24 (du 22 au 29 juin), 8, 7, 15 et 16 (du ler au 3 juin).

Change in the total number of adults issued from the larvae released., $A, B, C, D$ : eggplants 10, 11, 12 and 20 (18 and 19.05), 1 and 2 (9.06), 4, 5, 7, 9, 19, 21 and 24 (from 22 to 29.06), 7, 8, 15 and 16 (from 1 to 3.06 ).
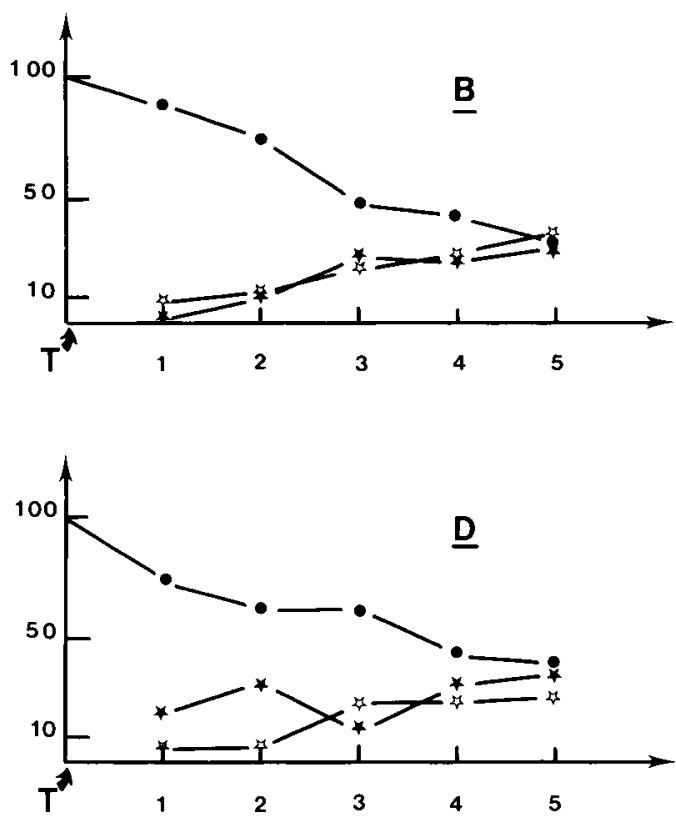

Change, relative to larvae released, in the population of S. undecimnotata larvae on treated $(\bullet)$ and neighbouring $(\star)$ eggplants. The remainder corresponds to missing larvae (k). $A, B, C, D$ : biological treatment (T) of egg-plants 1 and 2 (9.06), 5 and 6 (29.06), 5 (1.06) and 7 (23.06). 
Les parasites, essentiellement $A$. matricariae et Aphidius ervi Hal., sont apparus très tôt, dès l'installation des pucerons. Ils ont atteint un maximum de population au mois de juin (fig. 9).
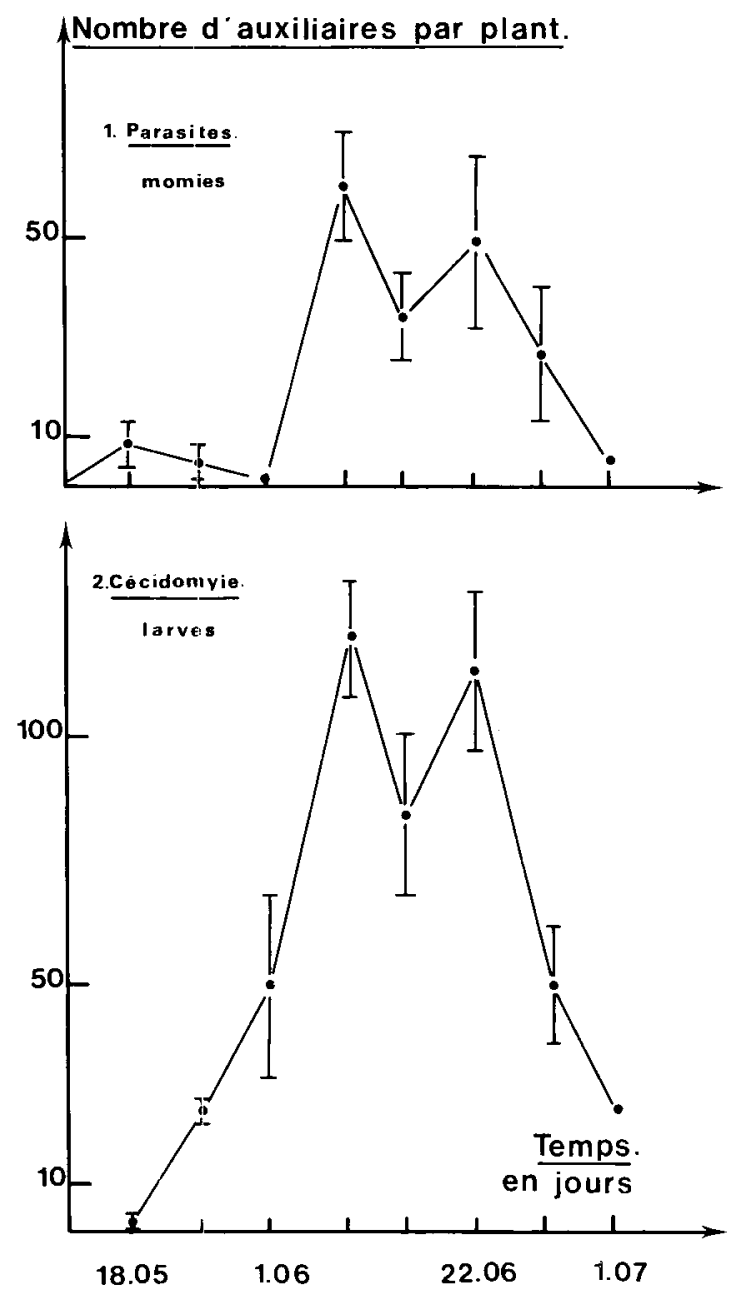

Figure 9

Variation du nombre moyen d'auxiliaires spontanés par aubergine : momies d'A. matricariae et A. ervi (parasites) et larves $d$ 'A. aphidimyza (Cecidomyie).

Change in the mean number of spontaneous aphidophagous insects per eggplant : mummies of A. matricariae and A. ervi (parasites) and larvae of $\mathrm{A}$. ajphidimyza (aphid midge).

Les larves de cécidomyie qui appartiennent à l'espèce $A$. aphidimyza, sont également très précoces et se rencontrent déjà dans les petites colonies des 3 pucerons présents dans cette serre. Leur efficacité doit pouvoir être quantifiée avec précision car les proies qu'elles tuent demeurent fixées au végétal par leurs pièces buccales tandis que leur corps, aplati et décoloré, reste dressé. La population larvaire a augmenté au cours du temps pour atteindre un maximum de $120 \pm 20$ individus par aubergine (fig. 9). Sur un certain nombre de plants ( $n^{\text {os }} 3,13,14,17$ et 18) qui portent respectivement $98,196,210,232$ et 331 larves, cette espèce est probablement responsable du blocage des populations aphidiennes et de l'absence d'intervention biologique avant la fin de l'expérimentation.

Sur 71 p. 100 des aubergines traitées avec les coccinelles, nous avons observé au contrôle suivant, une chute des populations de cette cécidomyie qui atteint, en moyenne, 54 p. 100 de l'effectif trouvé le jour des lâchers. Il est impossible de préciser si cette diminution résulte de leur destruction par la coccinelle ou plus simplement, de leur migration vers le sol en vue de leur transformation en pupe.

Par ailleurs, nous avons noté, à plusieurs reprises, la présence dans cette serre de quelques adultes de la coccinelle Coccinella septempunctata $\mathrm{L}$. et de larves de chrysope non identifiées.

\section{DISCUSSION}

Dans la majorité des publications antérieures (GURNEY \& HUSSEY, 1970 ; RAUTAPÄÄ, 1977 ; TULISALO \& TUOVINEN, 1975), le nombre de larves d'un lâcher est fixé, par approximations successives, en fonction de l'efficacité des traitements. La méthode de calcul de la taille de l'inoculum larvaire proposée dans ce travail, bien qu'étant empirique, constitue une approche nouvelle puisqu'elle est basée à la fois, sur les aptitudes trophiques des auxiliaires et sur l'évolution en biomasse des populations des proies.

Au moment des lâchers, le nombre moyen des pucerons par plant est de $3500 \mathrm{M}$. euphorbiae en 1980 et de $5600 M$. persicae en 1981. Le nombre moyen de larves placées sur chaque plant au cours de ces 2 années successives est respectivement de 51 et 80 (tabl. 3 et 4). Par conséquent, chaque coccinelle dispose de 68 (en 1980) et 71 (en 1981) proies lors de sa mise en place sur le végétal. Ces valeurs sont sensiblement supérieures à celles avancées par GURNEY \& HUSSEY (1970), une larve de coccinelle pour 20 aphides, et par RAUTAPÄÄ (1977), une larve de chrysope pour 50 pucerons. Elles sont par contre comparables à celles proposées par ADASHKEVICH \& KUZZNA (1974), une larve de Chrysopa septempunctata Wesmael. pour 50 à 100 proies.

Cette méthodologie, le choix de la coccinelle indigène $S$. undecimnotata qui fréquente la strate herbacée (IPERTI, 1965), l'utilisation des larves de dernier stade qui présentent une action immédiate et importante en raison de leurs aptitudes trophiques élevées, nous ont permis, dans la plupart des interventions biologiques, de réduire très substantiellement les populations des différents pucerons. Toutefois un certain nombre d'observations faites au cours de ces travaux semblent limiter l'emploi de cette espèce et, peut-être, mettre en cause ce type d'intervention biologique.

Le besoin général chez les prédateurs (HAGEN \& VAN DEN BoSCH, 1968) d'une quantité minimale de proies pour leur sédentarisation sur les végétaux constitue un critère capital dans le choix d'un auxiliaire. Compte tenu des difficultés d'élevage, il paraît préférable d'utiliser des espèces caractérisées par un seuil d'installation, le plus faible possible. Les chrysopes, que certains auteurs (LYON, comm. pers.) utilisent dès les premières apparitions des pucerons, et peutêtre certaines cécidomyies, d'après nos propres observations, paraissent à cet égard mieux armées que la coccinelle considérée. En effet, le seuil mis en évidence chez des larves de $S$. undecimnotata se situe très nettement après la période de colonisation des 
plantes par les aphides. De ce fait, il fait courir un risque à la culture, notamment à la production de fruits. Par ailleurs, il impose que la décision d'intervenir soit prise rapidement à la vue des résultats du dénombrement des proies. Cette méthode, qui est applicable sur un nombre limité de plants, paraît difficilement transposable dans de grandes serres car elle nécessite une technique très simple d'estimation des populations aphidiennes et une production importante et continue de cette coccinelle.

Le comportement des larves, en particulier leur stratification sur le végétal en harmonie avec celle des populations de pucerons, constitue également un critère de choix de l'auxiliaire. L'enlèvement des feuilles basales lorsque le végétal peut supporter ces mutilations (DELla GiUSTINA, 1970), peut constituer une méthode culturale complémentaire.

La mobilité des larves, qui peut être avantageuse dans la mesure où elle favorise leur dispersion sur l'ensemble de la plante, constitue un handicap lorsqu'elle se traduit par un gaspillage du matériel biologique.

$\mathrm{Si}$, comme le supposent certains auteurs, ces déplacements sont stimulés par " des effets de nombre ", ils peuvent mettre en cause la pratique des traitements inondatifs. Etant donné les potentialités des aphides, notamment leur aptitude à développer de nouvelles pullulations après des traitements inondatifs même efficaces, étant donné les difficultés actuelles de produire dans un laps de temps très court une biomasse considérable de coccinelles, il paraît plutôt souhaitable d'exercer à l'encontre de ces ravageurs une pression trophique continue destinée à maintenir leur population au-dessous d'un certain seuil de nuisibilité. Le comportement de fuite des adultes interdit à l'heure actuelle cette possibilité. La recherche d'espèces indigènes ou exotiques qui resteraient dans ce milieu, constitue une première voie d'approche (IPERTI, comm. pers.). La sélection de souches adaptées à ces conditions particulières ou éventuellement de souches caractérisées par une incapacité au vol peut être également envisagée.

Reçu le 9 mai 1983. Accepté le 19 décembre 1983.

\section{REMERCIEMENTS}

Nous remercions MM. PANis et RABASSE (I.N.R.A., Antibes) pour la détermination des insectes entomophages spontanés.

\section{RÉFÉRENCES BIBLIOGRAPHIQUES}

Adashkevich B. P., Kuzzna N. P., 1974. Chrysopids in vegetable crops. Zashch. Rast., 28-29.

Blackman R. L., 1967. The effects of different aphid foods on Adalia bipunctata L. and Coccinella 7 punctata L. Ann. Appl. Biol., 59, 207-219.

Della Giustina W., 1970. Etude sur la dynamique des populations d'insectes en serre maraîchère. Thèse, Paris.

Ferran A., Larroque M. M., 1977. Etude des relations proieprédateur : la consommation alimentaire et l'utilisation d'un puceron Myzus persicae Sulz. par les différents stades larvaires de la coccinelle Semiadalia undecimnotata Schn. (Col., Coccinellidae). Ann. Zool. Ecol. Anim., 9, 665-691.

Ferran A., Larroque M. M., 1979. Influence des facteurs abiotiques sur la physiologie alimentaire des larves de la coccinelle aphidiphage Semiadalia undecimnotata Schn. (Col., Coccinellidae). I. Action de la température. Entomophaga, 24, 403-410.

Frazer B. D., Gilbert N., Ives P. M., Raworth D. A., 1981. Predator reproduction and the overall predator-prey relationship. Can. Entomol., 113, 1015-1024.

Gurney B., Hussey N. N., 1970. Evaluation of some coccinellid species for the biological control of aphids in protected cropping. Ann. Appl. Biol., 65, 451-458.

Hagen K. S., Van Den Bosch R., 1968. Impact of pathogens, parasites and predators on aphids. Annu. Rev. Entomol., 13, 325-384.

Hämäläinen M., 1977. Control of aphids on sweet peppers, chysanthemums and roses in small greenhouses using the ladybeetles Coccinella septempunctata and Adalia bipunctata (Col. Coccinellidae). Ann. Agric. Fenn., 16, 117-131.

Hämäläinen M., 1980. Evaluation of two native coccinellids for aphid control in glasshouses. Bull. S.R.O.P./W.P.R.S., 111/3, 59-64.

Hassell M. P., Lawton J. H., Beddington J. R., 1976. The components of arthropod predation (of arthropod predation). I. The prey death-rate. J. Anim. Ecol., 45, 135-164.

Hussey N. W., Read W. H., Hesling J. J., 1969. The pest of protected cultivation. Edward Arnold ed., London, 87-96.

Hussey N. W., Bravenboer L., 1971. Control of pests in glass- house culture by the introduction of natural enemies. In Biological control, Huffaker C.B., ed., Plenum Press, New York, London, 195-216.

Iperti G., 1965. Some components of efficiency in aphidophagous coccinellids. Reprint from "Ecology of aphidophagous insects". Proc. Int. Symp. Liblice. Prague.

Ives P. M., 1981. Estimation of coccinellid number and movement in the field. Can. Entomol., 113, 981-997.

Kieckhefer R. W., Olson G. A., 1974. Dispersal of marked coccinellids from crops in South Dakota. J. Econ. Entomol., 67, 52-54.

Lyon J. P., 1976. Les populations aphidiennes en serre et leur limitation par l'utilisation expérimentale de divers entomophages. Bull. O.I.L.B./S.R.O.P., 1976/4, 64-76.

Lyon J. P., 1979. Lâchers expérimentaux de chrysopes et d'hyménoptères parasites sur pucerons en serre d'aubergines. Ann. Zool. Ecol. Anim., 11, 51-65.

Markkula M., Titanen K., Hämäläinen M., Forshberg A., 1979. The aphid midge Aphidoletes aphidimyza (Dip., Cecid.) and its use in the biological control of aphids. Ann. Entomol. Fenn., 45, 8998.

Murdoch W. W., Marks J. R., 1973. Predation by coccinellid beetles : experiments in switching. Ecology, 54, 160-167.

Onillon J. C., 1978. Modalités d'emploi des hyménoptères parasites dans la lutte contre les aleurodes. Bull. Tech. Inf., Ministère Agric., 332-333, 431-436.

Parr W. J., 1970. Glasshouse whitefly. Rep. Glasshouse Crops Res. Inst., 1969, 109-110.

Parr W. J., 1972. Biological control of glasshouse pests. J. RA.S.E., 133, 48-54.

Pralavorio M., 1978. Perspective de lutte biologique contre les tétranyques acariens nuisibles aux cultures sous serre. Bull. Tech. Inf. Ministère Agric., 332-333, 443-446.

Pralavorio M., Jourdheuil P., Millot P., 1975. Essais d'utilisation de l'acarien prédateur Phytoseiulus persimilis contre les tétranyques sur diverses cultures florales et maraîchères en serre. Ann. Zool. Ecol. Anim., 7, 211-226. 
Rabasse J. M., 1980. Dynamique des populations d'aphides sur aubergine en serre. I. Considérations générales sur la colonisation et le développement des populations de quatre espèces dans le sud de la France. Bull. S.R.O.P./W.P.R.S., 111/3, 187-198.

Rautapää J., 19:7. Evaluation of predator prey ratio using Chrysopa carnea Steph. in control of Rhopalosiphum padi L. Ann. Agric. Fenn., 16, 103-109.
Tamaki G., Weeks R. E., 1973. The impact of predators on populations of green peach aphids on field-grown sugar beets. Environ. Entomol., 2, 345-349.

Tulisalo U., Tuovinen T., 1975. The green lacewing Chrysopa carnea Steph. (Neuropt., Chrysopidae) used to control the green peach aphid Myzus persicae Sulz. and the potato aphid Macrosiphum euphorbiae Thom. (Homopt., Aphididae) on greenhouse green peppers. Ann. Entomol. Fenn., 41, 94-102. 